\title{
Emergency department visit volume variability
}

\section{Seung Woo Kang, Hyun Soo Park}

Department of Emergency Medicine, Jeju National University Hospital, Jeju, Korea

Objective One of the most important and basic variables in emergency department (ED) operations is patient visit volumes. This variable is usually predicted on the basis of the average ED patient visit volume over a certain period. However, ED patient visit variability is poorly understood. Therefore, we evaluated ED patient visit variability in order to determine if the average can be used to operate EDs.

Methods Nationwide ED patient visit data were from the standard emergency patient data of the National Emergency Department Information System. The data are transferred automatically by 141 EDs nationwide. The hourly ED visit volumes over 365 days were determined, and the variability was analyzed to evaluate the representativeness of the average.

Results A total of 4,672,275 patient visits were collected in 2013. The numbers of daily ED patient visits were widely dispersed and positively skewed rather than symmetric and narrow with a normal distribution.

Conclusion The daily variability of ED visit is too large and it did not show normal distribution. The average visit volume does not adequately represent ED operation.

Keywords Emergency service, hospital; Crowding; Efficiency; Management information systems

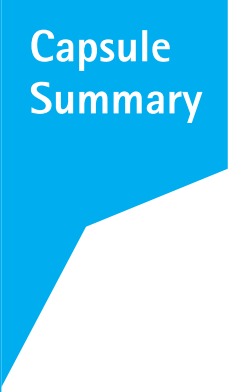

What is already known

To operate emergency department effectively, information on patient visits is important. Previously, the average emergency department patient visit volume was assumed to be a representative value.

What is new in the current study

We found that the average is not sufficient to describe the emergency department patient visit variability. More optimized parameters should be introduced for better analysis.
elSSN: 2383-4625

Received: 22 October 2014

Revised: 26 May 2015

Accepted: 2 June 2015

Correspondence to: Hyun Soo Park Department of Emergency Medicine, Jeju National University School of Medicine, 15 Aran 13-gil, Jeju 690-767, Korea E-mail:phs0331@gmail.com

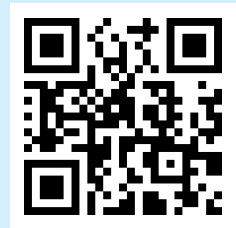

How to cite this article:

Kang SW, Park HS. Emergency department visit volume variability. Clin Exp Emerg Med 2015;2(3):150-154.

This is an Open Access article distributed under the terms of the Creative Commons Attribution Non-Commercial License (http:// creativecommons.org/licenses/by-nc/3.0/). 


\section{INTRODUCTION}

The maturation of emergency medicine as a specialty has coincided with dramatic increases in emergency department (ED) visit rates worldwide. ${ }^{1}$ Patients who present to EDs often face long wait times for treatment; moreover, those who require admission wait even longer for an inpatient hospital bed. ${ }^{2}$ ED timeliness is a critical factor affecting the operational performance of the complex processes required to deliver emergency care. Operational performance ultimately affects the quality of care, patient safety, and operational and financial efficiency. ${ }^{3}$ Many interventions to manage ED crowding have been implemented in order to improve the efficiency of ED operation by eliminating waste, reducing variability, and increasing flexibility. ${ }^{4,5}$ The problem remains despite these efforts, because ED crowding is not only a problem related with individual EDs, but is rather a reflection of a larger supply and demand mismatch in the healthcare system. ${ }^{2}$

In the conceptual model of the ED, previous interventions mainly target throughput and output components. ED supply systems concerning patient care processes and patient disposition are manageable; however, the input component, which includes any condition, event, or system characteristic that contributes to the demand for ED services, is not easily reducible or predictable. ${ }^{2}$ Ambulance diversion to other EDs or telephone triage services to reduce ED demand have limited effects on the overall emergency medical system. ${ }^{3}$ Furthermore, ED demand at a given time appears impossible to predict, and there is a lack of analytic research. The input component usually predicts the average ED patient volume over a certain period, despite the lack of evidence indicating that the average is adequately representative of the variability of ED patient visits. ${ }^{6-8}$

Efficient operation requires individual EDs to estimate how many busy days will occur and can be reduced with additional medical resources as well as how often "on-call" resources will be needed. Thus, hourly ED visit data should be collected and processed to address these questions. Accordingly, processed nationwide ED visit data could be used to construct a road map to develop policies for the entire healthcare system from the perspective of the expanded input-throughput-output conceptual model. ${ }^{9}$ Thus, the present study evaluated the daily variability in hourly ED visit volume.

\section{METHODS}

\section{Study design and primary outcome}

This was a retrospective observational study that aimed to characterize the daily variability of hourly ED visits and evaluate the representativeness of the average. We created and analyzed 24 bar graphs in a histogram of the average hourly ED visit volumes over 365 days as well as 24 box-plots of hourly ED visit volume over 365 days.

We analyzed the nationwide ED patient visit time data of the National Emergency Medical Center not only to provide information about a particular ED, but also to evaluate EDs in general. Since 2004, the National Emergency Medical Center in Korea has collected standard ED visit patient data including time of visit, sex, age, chief complaint, result of disposition, route of visit, vital signs, and date and time of discharge from nationwide EDs via an automated reporting system, the National Emergency Department Information System (NEDIS); it offers these data as a text file containing the times of visit of nationwide EDs in 2013. ${ }^{10}$

\section{Data analysis}

The ED visit patient data were divided into 24 hours and 365 days according to visit time, and a $365 \times 24$ data table was constructed. The 8,760 values in the resultant data table were analyzed by descriptive statistics to create bar graphs showing the means and 95\% confidence intervals as well as box plots to present the hourly and daily characteristics of ED visit variability for comparison with the bar graphs. For the statistical analysis of the representativeness of the means, the Shapiro-Wilk test was performed to test the normality of the distribution of the hourly ED visit volume over 365 days; skewness and kurtosis were also calculated.

The text file including ED visit time data were collected and converted to comma separated value format by using Microsoft Excel 2010 (Microsoft Corp., Redmond, WA, USA). The graphs were created and statistical analysis was performed by using $R$ version 3.1.3 developed by the R Foundation for Statistical Computing (R Development Core Team, 2005, http://www.R-project. org). The study protocol and exemption from informed consent were approved by the institutional review board of the Jeju national University Hospital.

\section{RESULTS}

In 2013, the NEDIS annual report stated 4,919,181 patients visited 141 EDs in 16 provinces nationwide (Table 1). ${ }^{10}$ However, as the information transmission rate to the NEDIS was approximately $94 \%$, the available text file contained the arrival times of 4,672,275 patients in 2013. ${ }^{10}$

The bar graphs and box plots of EDs nationwide are shown in Figs. 1 and 2, respectively. The hourly visit pattern in one day showed a bimodal distribution with peaks from 10:00 to 11:00 and 20:00 to 21:00. The period with the lowest visits was 02:00 to 
Table 1. Patient visit volumes of EDs nationwide in 2013

\begin{tabular}{lrrr}
\hline Province & Population & Studied ED count & ED visit volume \\
\hline A & $10,143,645$ & 30 & $1,287,020$ \\
B & $3,527,635$ & 8 & 218,802 \\
C & $2,501,588$ & 5 & 199,492 \\
D & $2,879,782$ & 7 & 294,589 \\
E & $1,472,910$ & 6 & 127,455 \\
F & $1,532,811$ & 5 & 191,596 \\
G & $1,156,480$ & 2 & 72,835 \\
H & $12,234,630$ & 29 & $1,147,841$ \\
I & $1,542,263$ & 6 & 163,565 \\
J & $1,572,732$ & 4 & 119,577 \\
K & $2,047,631$ & 6 & 198,958 \\
L & $1,872,965$ & 6 & 148,868 \\
M & $1,907,172$ & 5 & 108,036 \\
N & $2,699,440$ & 10 & 275,902 \\
O & $3,333,820$ & 7 & 238,221 \\
P & 593,806 & 5 & 126,424 \\
Total & $51,019,310$ & 141 & $4,919,181$ \\
\hline
\end{tabular}

Data from National Emergency Medical Center. Annual report 2013 [Internet]. Seoul: National Emergency Medical Center; 2014. ${ }^{10}$

$E D$, emergency department.

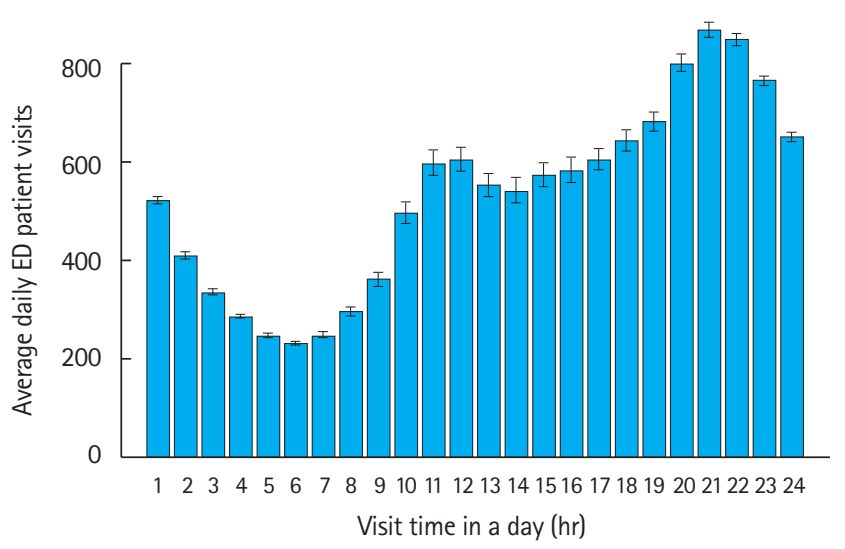

Fig. 1. The average hourly visit volume with 95\% confidence intervals of nationwide emergency department (ED) according to visit times in 2013.

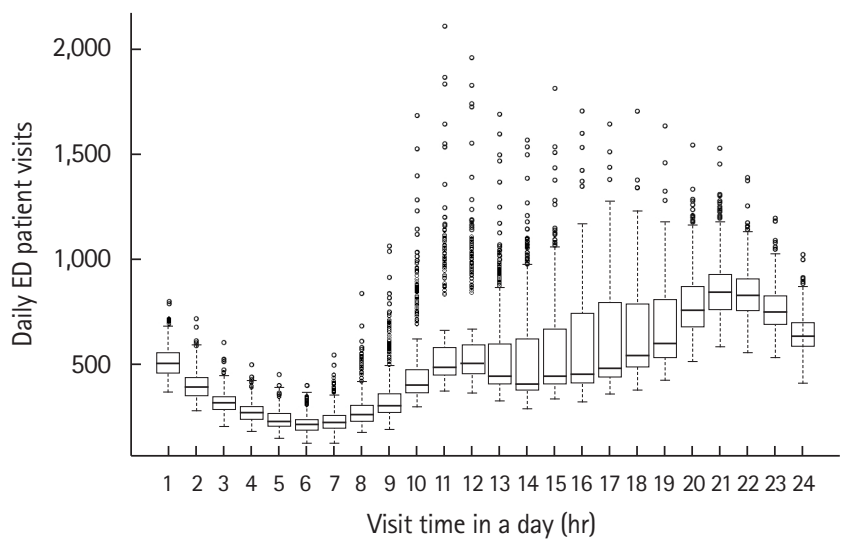

Fig. 2. Visit times of nationwide emergency departments (EDs) in 2013. The daily variation of hourly visits in a year of nationwide EDs.
08:00. The box plots (Fig. 2) of the ED visit volume at each time showed upside-skewed interquartile ranges rather than a symmetric distribution in addition to the median volume.

In contrast to the nationwide ED graph, the single ED graphs showed a similar bimodal distribution of hourly visit volume in one day. However, the skewness and kurtosis of box plots of single EDs were smaller than those of nationwide EDs (Figs. 3, 4).

Statistically, the inter-day difference of hourly visit volumes was too large to assume a normal distribution despite the large dataset $(\mathrm{P}<0.05)$. Furthermore, both the nationwide and individual ED data were right skewed (upside skewed) and widely dispersed (Tables 2, 3).

\section{DISCUSSION}

The bimodal feature of the average hourly ED patient visit volumes identified in the present study is similar to the findings of previous studies. ${ }^{11,12}$ The authors of those studies assumed there was no

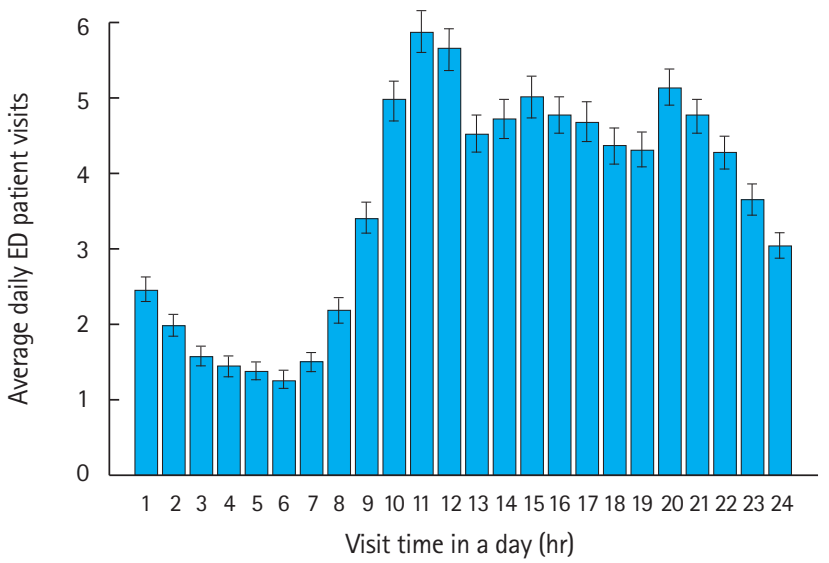

Fig. 3. The average hourly visit volume with 95\% confidence intervals of one emergency department (ED) according to visit times in 2013.

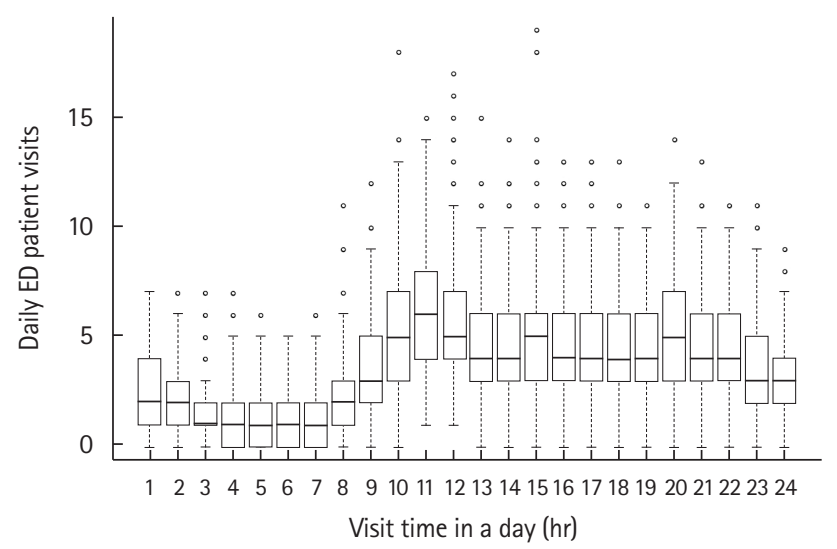

Fig. 4. Visit times of individual emergency departments (EDs) in 2013. The daily variation of hourly visits in a year of one ED (2013). 
Table 2. Skewness, kurtosis, and normality of the distribution of hourly nationwide emergency department patient visit volumes

\begin{tabular}{cllcl}
\hline Time & Skewness & Kurtosis & Shapiro-Wilk test & P-value \\
\hline 1 & 0.78042 & 3.797448 & 0.960829 & $<0.05$ \\
2 & 1.044187 & 4.778892 & 0.944757 & $<0.05$ \\
3 & 0.975646 & 4.765419 & 0.951092 & $<0.05$ \\
4 & 0.936456 & 4.13346 & 0.949906 & $<0.05$ \\
5 & 0.982596 & 4.711342 & 0.951594 & $<0.05$ \\
6 & 1.045561 & 4.24744 & 0.933705 & $<0.05$ \\
7 & 1.586856 & 6.237132 & 0.874597 & $<0.05$ \\
8 & 2.085974 & 8.66038 & 0.798833 & $<0.05$ \\
9 & 2.015533 & 7.143919 & 0.744704 & $<0.05$ \\
10 & 2.146164 & 7.867818 & 0.690205 & $<0.05$ \\
11 & 2.387513 & 9.595485 & 0.663227 & $<0.05$ \\
12 & 2.266942 & 8.834264 & 0.69089 & $<0.05$ \\
13 & 1.932458 & 6.932239 & 0.741465 & $<0.05$ \\
14 & 1.616581 & 4.893624 & 0.745781 & $<0.05$ \\
15 & 1.666196 & 5.403175 & 0.745152 & $<0.05$ \\
16 & 1.513102 & 4.848528 & 0.767458 & $<0.05$ \\
17 & 1.356479 & 4.330698 & 0.790227 & $<0.05$ \\
18 & 1.335169 & 4.499183 & 0.829125 & $<0.05$ \\
19 & 1.252993 & 4.133825 & 0.849254 & $<0.05$ \\
20 & 1.083121 & 3.95945 & 0.917805 & $<0.05$ \\
21 & 1.022187 & 4.117709 & 0.934925 & $<0.05$ \\
22 & 0.847581 & 4.056204 & 0.959112 & $<0.05$ \\
23 & 0.861803 & 3.970081 & 0.957433 & $<0.05$ \\
24 & 0.715088 & 3.916872 & 0.972531 & $<0.05$ \\
\hline & & & &
\end{tabular}

significant variability in visits over 365 days at a given time of day; therefore, the average was used as the representative value. However, the box plots showed positively skewed and widely dispersed distributions of visit times and this causes the more-frequent "low visit volume days" to decrease the average, consequently masking the less-frequent "high visit volume days." In the case of a given ED with a visit volume similar to that shown in Fig. 4 (with a volume difference of approximately 1.6 patients per hour between the 1st quartile and average), an ED operator who prepares the ED expecting the average ED visit volume may experience 1.6 more patients per hour in three months (25\%) in a given year. Unfortunately, the unbounded positive range also indicates that ED crowding cannot be completely prevented in cases such as disasters. Therefore, "permissive busy ED days" should be determined in order to prevent excessive waste of medical resources. Accordingly, further simulation studies will allow us to predict how much more medical resources might be needed to reduce the number of busy days below a particular percent in a year.

The features of the box plots presented herein may differ from those of other emergency medical systems with different socioeconomic situations and emergency medical service utilization habits. Furthermore, as patient visit volumes can change over time, the
Table 3. Skewness, kurtosis, and normality of the distribution of hourly patient visit volumes at individual emergency departments

\begin{tabular}{cllcl}
\hline Time & Skewness & Kurtosis & Shapiro-Wilk test & P-value \\
\hline 1 & 0.504972 & 2.623897 & 0.934153 & $<0.05$ \\
2 & 0.344041 & 2.691325 & 0.927917 & $<0.05$ \\
3 & 0.909751 & 4.048702 & 0.888703 & $<0.05$ \\
4 & 1.067029 & 4.342416 & 0.865747 & $<0.05$ \\
5 & 0.891327 & 3.653524 & 0.875443 & $<0.05$ \\
6 & 0.831205 & 3.158926 & 0.863741 & $<0.05$ \\
7 & 0.706727 & 2.943521 & 0.890265 & $<0.05$ \\
8 & 1.257117 & 5.726697 & 0.888624 & $<0.05$ \\
9 & 0.754747 & 3.989155 & 0.946088 & $<0.05$ \\
10 & 0.81667 & 4.631885 & 0.950402 & $<0.05$ \\
11 & 0.554688 & 3.130892 & 0.965915 & $<0.05$ \\
12 & 0.770079 & 4.317239 & 0.95373 & $<0.05$ \\
13 & 0.884146 & 4.757388 & 0.945048 & $<0.05$ \\
14 & 0.574378 & 3.171003 & 0.963394 & $<0.05$ \\
15 & 1.257715 & 6.201206 & 0.918434 & $<0.05$ \\
16 & 0.624008 & 3.061558 & 0.956064 & $<0.05$ \\
17 & 0.801788 & 3.676728 & 0.944638 & $<0.05$ \\
18 & 0.635857 & 3.299813 & 0.957029 & $<0.05$ \\
19 & 0.584441 & 2.880888 & 0.953532 & $<0.05$ \\
20 & 0.543809 & 3.340312 & 0.966608 & $<0.05$ \\
21 & 0.689478 & 3.306855 & 0.951212 & $<0.05$ \\
22 & 0.358093 & 2.939101 & 0.968227 & $<0.05$ \\
23 & 0.592459 & 3.352529 & 0.95641 & $<0.05$ \\
24 & 0.5825 & 3.015797 & 0.944147 & $<0.05$ \\
\hline & & & &
\end{tabular}

annual change should be taken into consideration. ${ }^{1}$ Nevertheless, the box plots verify the daily variability of ED visit volumes.

\section{CONFLICT OF INTEREST}

No potential conflict of interest relevant to this article was reported.

\section{ACKNOWLEDGMENTS}

This work was supported by a research grant from Jeju National University Hospital in 2013.

\section{REFERENCES}

1. Pines JM, Hilton JA, Weber E, et al. International perspectives on emergency department crowding. Acad Emerg Med 2011; 18:1358-70.

2. Asplin BR, Magid DJ, Rhodes KV, Solberg LI, Lurie N, Camargo CA Jr. A conceptual model of emergency department crowding. Ann Emerg Med 2003;42:173-80.

3. Handel $D$, Epstein $S$, Khare $R$, et al. Interventions to improve 
the timeliness of emergency care. Acad Emerg Med 2011;18: 1295-302.

4. Pines JM, McCarthy ML. Executive summary: interventions to improve quality in the crowded emergency department. Acad Emerg Med 2011;18:1229-33.

5. Soremekun OA, Terwiesch C, Pines JM. Emergency medicine: an operations management view. Acad Emerg Med 2011;18: 1262-8.

6. Connelly LG, Bair AE. Discrete event simulation of emergency department activity: a platform for system-level operations research. Acad Emerg Med 2004;11:1177-85.

7. Su $Y$, Huang $X$, Jin Z. Modeling emergency service centers with simulation and system dynamics. In: UK Society for Modelling and Simulation. 2009 UKSIM'09 11th International Conference on Computer Modelling and Simulation; 2009 Mar 25-7; Cambridge: UK. [place unknown]: IEEE; 2009. p. 305-10.

8. Yeh JY, Lin WS. Using simulation technique and genetic algorithm to improve the quality care of a hospital emergency department. Expert Syst Appl 2007;32:1073-83.

9. Kamal N, Barnard DK, Christenson JM, et al. Addressing emergency department overcrowding through a systems approach using big data research. J Health Med Inform 2014. DOI: http://dx.doi.org/10.4172/2157-7420.1000148.

10. National Emergency Medical Center. Annual report 2013 [Internet]. Seoul: National Emergency Medical Center; 2014 [cited 2015 Jul 1]. Available from: http://www.nemc.or.kr/egen/ inf.emb13.do.

11. Ahmed MA, Alkhamis TM. Simulation optimization for an emergency department healthcare unit in Kuwait. Eur J Oper Res 2009;198:936-42.

12. Eskandari $H$, Riyahifard M, Khosravi $S$, Geiger CD. Improving the emergency department performance using simulation and MCDM methods. In: Winter Simulation Conference. Proceedings of the 2011 Winter Simulation Conference (WSC); 2011 Dec 11-4; Phoenix, AZ, USA. [place unknown]: IEEE; 2011. p. 1211-22. 